\title{
Energetic Convergence of a New Hybrid Mixed Finite Element
}

\author{
M.R.T. Arruda ${ }^{a}$, P.F.T. Arruda ${ }^{b}$ and B.J.F. Lopes ${ }^{b}$
}

${ }^{a}$ CERIS-Civil Engineering Research and Innovation for Sustainability, Instituto Superior Técnico, Universidade de Lisboa, Av. Rovisco Pais, 1 1049-001 Lisboa, Portugal

${ }^{b}$ Instituto Superior Técnic, Universidade de Lisboa, Av. Rovisco Pais, 1 1049-001 Lisboa, Portugal

\begin{tabular}{l}
\hline A R T I C L EI N F O \\
\hline Article history: \\
Received 4 June, 2018 \\
Accepted 14 July 2019 \\
Available online \\
14 July 2019 \\
\hline Keywords: \\
Hybrid-mixed formulation \\
Stress model, Legendre \\
polynomials \\
Elastic Strain Energy \\
Parametric Convergence
\end{tabular}
\begin{abstract}
A B S T R A C T
This paper presents a new hybrid double mixed stress (4f-HMS) model, for the static analysis of isotropic plane structures, in which it is assumed a physically and geometrically linear behaviour. The main improvement of this model is, it approximates independently the three most important fields in the domain, more specifically the strain field, the stress field and the displacement field of each element. The displacements along the static boundary, considered to include inter-element boundaries, are also directly approximated. For the approximation functions in the space domain, a complete set of orthonormal Legendre polynomials are used. The adoption of these functions enables the use of analytical closed form solutions, for the computation of all linear structural operators, and leads to the development of very effective p- refinement procedures. The model being discussed is tested in terms of convergence capabilities using classical elastic strain energy and other common variables, in which the monotonic convergence is tested. To validate the model and to illustrate its potential, several numerical examples are discussed and comparisons are made, with solutions obtained using analytical results and other known finite element formulations.
\end{abstract}

\section{Introduction}

In the computational mechanics field, the most popular method for solving non-linear structural problems is the Finite Element Method (Hughes, 2003). This method is still nowadays the most popular numerical technique used in the field of continuum mechanics, due to its explicit physical meaning (Ghali et al., 1997), its theoretical robustness and efficiency (Clough et al., 1999). In spite of the popularity, and computational robustness of the conventional finite element method (CFE), this formulation still presents some drawbacks (Bathe, 1996) and some unfixed problems (Zienkiewicz, 2000). For this reason, several alternative formulations have been proposed over the years, such as: hybrid stress (Cen et al., 2011a; Cen et al., 2011b; Pian et al., 1969), hybrid displacement (Veubeke, 1965), Trefftz elements (Jirousek et al., 1977; Ruoff, 1973; Stein, 1973), the extended finite element method (XFEM) (Moes et al., 1999), isogeometric finite elements (Cottrell et al,. 2009) and meshfree methods (Liu, 2003). It is possible to summarize three main classes of hybrid mixed finite element formulation, namely the hybrid-mixed, the hybrid and the hybrid- Trefftz formulations. These first studies, using alternative approximation functions with non-conventional methods, were conducted by Freitas et al. (1996) during the late nineties. Two models are derived for each formulation, the displacement and the stress models. All formulations evolve

* Corresponding author.

E-mail addresses: mario.rui.arruda@tecnico.ulisboa.pt (M.R.T. Arruda) 
directly from the first principles of mechanics, in particular equilibrium, compatibility and constitutive relations. What distinguishes the three types of formulations, is the set of constraints enforced a priori, on the domain approximations.

Since the late 80s the Structural Analysis Research Group of Instituto Superior Técnico has been involved in the development of alternatives for hybrid and mixed finite element formulations. Although these formulations present some advantages, they still have some limitations (Freitas et al., 1999). In recent years, hybrid-mixed stress finite element models have been developed for the static analysis of plane stretching and plate bending problems (Castro et al., 2006; Castro et al., 2001; Pereira et al., 1996a; Pereira et al., 2000). After establishing the properties of these alternative formulations, the next challenge was to extend their application to the analysis of problems closer to engineering practice. In a first step, elastoplastic models for the analysis of plane structures have been developed. After that, continuum damage models for the physically non-linear analysis of concrete structures have been studied and implemented (Silva et al., 2006b).

One of the main advantages associated with the use of this type of formulation is the flexibility introduced in the selection of the approximation functions. This fact makes it possible to use functions with special properties that cannot be implemented in the framework of conventional finite element models, such as Legendre polynomials, systems of wavelets and Walsh series. The hybrid-mixed stress models may also lead to quasi-equilibrated solutions which are very suitable for design purposes. It is then possible to define the main advantages of hybrid mixed stress formulations:

- There is a great flexibility in choosing the approximation functions (Freitas et al., 1999).

- The right choice for the degrees of the approximation functions may lead to "quasi-equilibrated" solutions (Arruda et al., 2011b).

- If the approximation functions are orthogonal, the governing system is sparse (Arruda, 2011).

- In the hybrid mixed stress (HMS) model, more specifically the mixed formulations, the deformation field is separated from the stress field. If the degrees are correctly chosen then the shear locking does not occur (Malkus et al., 1978).

- The $p$ - refinement is effective and easy to implement, and in linear analysis all operators of the governing system are computed analytically. No numerical integration is needed in linear analysis (Pereira et al., 1996a).

- For simple geometries the use of macro-elements avoids the use of mesh generators (Arruda et al., 2013b).

- In non-linear analysis, the evolution of the constitutive relation depends directly on the stress field. Since the HMS model directly approximates the stress field, some advantages may be obtained (Arruda et al., 2013a).

Due to the type of enforcement followed, for the equilibrium and compatibility conditions in the domain, and because the connection between elements is ensured by the weighted residual enforcement of the equilibrium conditions at the common boundary, the presented formulation is considered to lead to a stress model.

\section{Presentation of the $4 \mathrm{f}-\mathrm{HMS}$}

A new Hybrid Double Mixed Stress (4f-HMS) element that also approximates the deformation field (Arruda et al., 2011a; Arruda et al., 2013a), is presented and tested. This element tends to overcome the known difficulties, when using damage mechanics with HMS (Silva et al., 2006a), for governing systems based on flexibilities. The finite element models presented in this work, are based on the use of orthonormal Legendre polynomials as approximation functions. The properties of these functions, allow for definition of analytical closed form solutions, for the computation of all structural operators in linear 
analysis. Numerical integration schemes are thus completely avoided. The numerical stability, associated with the use of Legendre polynomial bases, enables the use of macro-element meshes, where the definition of highly effective $p$-adaptive refinement procedures is simplified. A detailed presentation of these functions can be found in (Mendes et al., 2009).

\section{Research Significance and Objectives}

Isotropic elastic constitutive relations are applied, in order to study the monotonic convergence of the strain energy with the 4f-HMS for 2D plane structures. These 2D tests are fundamental and sufficient, to study the convergence of strain energy in hybrid mixed formulation, as pointed out by Castro (Castro 2011). Contrary to conventional FEM, there is no guarantee in the monotonic convergence of Hybrid Mixed formulation, as pointed out by other authors (Pereira et al., 1996b; Santos, 2009). Therefore, numerical tests are necessary to suppress these questions, when a new hybrid mixed model is presented. This is the first energetic test applied to study the numerical convergence with the $4 \mathrm{f}-\mathrm{HMS}$, which will fill this gap in the scientific knowledge. To validate the model being proposed, to illustrate its potential and to assess its accuracy (and numerical efficiency), several numerical examples are discussed and comparisons are made with solutions provided by analytical solutions from other authors.

\section{Previous Work and Studies with the 4f-HMS}

Although new, this model has already been studied and applied in structural non-linear analysis. The first studies, concerned only the static non-linear analysis of concrete structures, using continuum damage mechanics (Arruda et al., 2013a), with the damage model of Comi (Comi et al., 2001b) and non-local regularization (Comi et al., 2001a), suppressing CFE in terms of total number of iterations. The next study tested the dynamic non-linear analysis of concrete dams, using once again the 4f-HMS (Arruda et al., 2013b), with the damage model of Mazars (Mazar,s 1984). In all numerical simulations, there is a good agreement with the experimental results and numerical results presented by CFE from other authors. When using the 4f-HMS model in non-linear analysis, the discretizations, can lead to systems with a relatively large number of degrees of freedom. However, as such systems are always very sparse, the use of algorithms specially designed for the storage and processing of sparse systems of large dimensions, ensures the effectiveness of the numerical computation process.

\section{Fundamental Relations}

Consider a set of static forces, acting in an elastic body. The equilibrium, compatibility and elasticity equations governing the behaviour of that structure may be expressed as (Silva et al. 2006a):

$$
\begin{aligned}
& D s+b=0 \\
& e=D^{*} u \\
& s=k e
\end{aligned}
$$

The vectors $s, e$ and $u$ list the independent components of the stress, strain and displacement fields, respectively. Assuming a geometrically linear behaviour, the differential equilibrium operator $D$, and the differential compatibility operator $D^{*}$, represent linear and adjoint operators. The vector $b$ lists the body force components. The boundary of the structure may be subdivided into two complementary regions: the kinematic boundary, $\Gamma_{u}$, in which the value for the displacement fields is prescribed and the static boundary, $\Gamma_{t}$, where the applied forces are prescribed. It can be written:

$$
\begin{aligned}
& u=u_{\Gamma} \text { on } \Gamma_{u} \\
& N s=t \text { on } \Gamma_{t}
\end{aligned}
$$


The vectors $u_{\Gamma}$ and $t$, gather the components of the prescribed displacements and forces, on the kinematic and static boundaries, respectively. The matrix $N$, lists the components of the unit outward normal vector. For a plane element, the operators and the vectors take the form of Eq. (6), Eq. (7) and in plane stress the stiffness is defined as Eq. (8).

$$
\begin{array}{ll}
D=\left[\begin{array}{ccc}
\frac{\partial}{\partial x} & 0 & \frac{\partial}{\partial y} \\
0 & \frac{\partial}{\partial y} & \frac{\partial}{\partial x}
\end{array}\right] \quad s=\left[\begin{array}{c}
\sigma_{x} \\
\sigma_{y} \\
\sigma_{x y}
\end{array}\right] \quad b=\left[\begin{array}{l}
b_{x} \\
b_{y}
\end{array}\right] \quad u=\left[\begin{array}{l}
u_{x} \\
u_{y}
\end{array}\right] \\
N=\left[\begin{array}{ccc}
n_{x} & 0 & n_{y} \\
0 & n_{y} & n_{x}
\end{array}\right] \quad e=\left[\begin{array}{c}
\varepsilon_{x} \\
\varepsilon_{y} \\
\gamma_{x y}
\end{array}\right] \quad t=\left[\begin{array}{l}
t_{x} \\
t_{y}
\end{array}\right] \quad u_{\Gamma}=\left[\begin{array}{l}
u_{\Gamma x} \\
u_{\Gamma y}
\end{array}\right] \\
D^{*}=\left[\begin{array}{cc}
\frac{\partial}{\partial x} & 0 \\
0 & \frac{\partial}{\partial y} \\
\partial & \partial
\end{array}\right] \quad k=\frac{E}{\left(1-v^{2}\right)}\left[\begin{array}{lll}
1 & v & 0 \\
v & 1 & 0 \\
0 & 0 & (1-v) / 2
\end{array}\right]
\end{array}
$$

\section{Hybrid Double Mixed Stress Model}

The hybrid double mixed stress (4f-HMS) model presented in this work, considers three independent approximations for the stress, strain and displacement fields in the domain of each element, expressed as follows.

$$
\begin{aligned}
& s=S_{V} X_{V} \text { in } V \\
& u=U_{V} q_{V} \text { in } V \\
& e=E_{V} \epsilon_{V} \text { in } V
\end{aligned}
$$

Matrices $S_{V}, E_{V}$ and $U_{V}$, gather the approximation functions and vectors $X_{V}, \epsilon_{V}$ and $q_{V}$, list the corresponding weights. The displacement field along the static boundary (which includes the boundaries between neighbouring elements) is also approximated:

$$
u=U_{\Gamma} q_{\Gamma} \text { on } \Gamma_{t}
$$

The matrix $U_{\Gamma}$, lists the approximation functions and vector $q_{\Gamma}$ the corresponding weights. The equilibrium condition in the domain is enforced, using the following weighted residual form (Pereira et al. 1996b):

$$
\int U_{V}^{t}(D s+b) d V=0 \text {. }
$$

Replacing in Eq. (11), the approximations defined for the stress fields in the domain Eq. (9), it is possible to recover the domain equilibrium condition in the discrete model, expressed as:

with

$$
A_{V}^{t} X_{V}=-Q_{V}
$$




$$
A_{V}^{t}=\int U_{V}^{t} D S_{V} d V \quad Q_{V}=\int U_{V}^{t} b d V
$$

The equilibrium conditions on the boundary presented in Eq. (14), the compatibility conditions in the domain displayed in Eq. (17) and the constitutive Eq. (20), are all also enforced in a weighted residual form (Arruda et al., 2011b). Assuming for the moment, a linear elastic behaviour it is possible to write:

$$
\int U_{\Gamma}^{t}(N s-t) d \Gamma_{t}=0
$$

leading to:

$$
A_{\Gamma}^{t} X_{V}=Q_{\Gamma}
$$

with

$$
\begin{gathered}
A_{\Gamma}^{t}=\int U_{\Gamma}^{t}\left(N S_{V}\right) d \Gamma_{t} \\
Q_{\Gamma}=\int U_{\Gamma}^{t} t d \Gamma_{t} \\
\int S_{V}^{t}\left(e-D^{*} u\right) d V=0
\end{gathered}
$$

resulting in:

$$
B_{V}^{t} \epsilon_{V}=-A_{V} q_{V}+A_{\Gamma} q_{\Gamma}+\bar{u}_{\Gamma}
$$

with

$$
\begin{array}{r}
\bar{u}_{\Gamma}=\int\left(N S_{V}\right)^{t} u_{\Gamma} d \Gamma_{u} \\
B_{V}^{t}=\int S_{V}^{t} E_{V} d V \\
\int E_{V}^{t}(k e-s) d V=0
\end{array}
$$

leads to:

$$
B_{V} X_{V}=\mathbb{K} \epsilon_{V}
$$

with

$$
\mathbb{K}=\int E_{V}^{t} k E_{V} d V
$$

The governing system Eq. (23) is obtained by combining the equilibrium, compatibility and elasticity conditions in the discrete model.

$$
\left[\begin{array}{cccc}
-\mathbb{K} & B_{V} & 0 & 0 \\
B_{V}^{t} & 0 & -A_{\Gamma} & A_{V} \\
0 & -A_{\Gamma}^{t} & 0 & 0 \\
0 & A_{V}^{t} & 0 & 0
\end{array}\right]\left\{\begin{array}{c}
\epsilon_{V} \\
X_{V} \\
q_{\Gamma} \\
q_{V}
\end{array}\right\}=\left\{\begin{array}{c}
0 \\
\bar{u}_{\Gamma} \\
-Q_{\Gamma} \\
-Q_{V}
\end{array}\right\}
$$


The elastic strain energy, is given by Eq. (24) as referred in (Holzapfel, 2000). In this case, it is possible to replace the approximations adopted in Eq. (9), obtaining Eq. (24) and deduce the elastic strain energy for the 4f-HMS, using isotropic constitutive relations.

$$
\begin{aligned}
& \Pi_{e}=\frac{1}{2} \int s^{t} e d V \\
& \Rightarrow \Pi_{e}=\frac{1}{2} \int\left(S_{V} X_{V}\right)^{t} E_{V} \epsilon_{V} d V \\
& \Rightarrow \Pi_{e}=\frac{1}{2} X_{V}^{t} B_{V}^{t} \epsilon_{V}
\end{aligned}
$$

The elastic strain energy in conventional finite element formulation (CFE), is only function of the node displacement and stiffness matrix (Cook et al., 2002), contrary to hybrid mixed formulation.

$$
\Pi_{e}^{C F E}=\frac{1}{2} q^{t} K q
$$

The relationship between the degrees of the polynomials, used for approximation of the stress fields and degrees of the polynomials associated with the displacement field, should be carefully established to avoid the appearance of spurious modes, which are manifested through the existence of dependencies in the governing system. In this case, if $n$ is used for the stress and strain field, then $n-1$ will be used for the displacement field, in order to avoid the referred dependencies (Castro et al., 2006; Hughes, 2003; Zienkiewicz et al., 2000).

In this work, equilibrium finite elements proposed by (De Almeida et al., 1991; Freitas, 1990; Pereira, 1996), more specifically hybrid stress (HS) elements are also used in some examples, namely the cantilever benchmark. More details on the governing system and its variables can be found in the previously cited works, in which it is demonstrated that the governing system is assembled as displayed in Eq. (27), and the strain energy is given by Eq. (28). The vector $X_{V}$, is the same as Eq. (9), and stands for the weights of the approximation functions, that guarantee the equilibrium in the domain $\left(D S_{V} X_{V}=\right.$ 0 ), based on the Pascal triangle. The vector $q_{\Gamma}$, has the same assumption as described in Eq. (10), which is related to the boundary displacement in each side of the finite element. The independent terms $\bar{u}_{\Gamma}$ and $Q_{\Gamma}$, are the prescribed displacement and applied forces in the kinematic and static boundary respectively. The operator $A_{\Gamma}$, is identical to Eq. (16), but the flexibility operator $\mathbb{F}$, is constructed using the approximation function for the stress field in the domain (Freitas et al., 1993). This last term is given by Eq. (26).

$$
\begin{aligned}
& \mathbb{F}=\int S_{V}^{t} k^{-1} S_{V} d V, \\
& {\left[\begin{array}{cc}
\mathbb{F} & -A_{\Gamma} \\
-A_{\Gamma} & 0
\end{array}\right]\left\{\begin{array}{l}
X_{V} \\
q_{\Gamma}
\end{array}\right\}=\left\{\begin{array}{c}
\bar{u}_{\Gamma} \\
-Q_{\Gamma}
\end{array}\right\},} \\
& \Pi_{e}^{H S}=\frac{1}{2} S_{V}^{t} \mathbb{F} S_{V} .
\end{aligned}
$$

Using a displacement and a stress formulation of finite element, it is possible to calculate an upper and lower bound of the energy error and therefore, estimate the rate of convergence of the 4f-HMS in the numerical examples.

\section{Approximation Functions}

The use of hybrid and hybrid-mixed finite element models, based on the use of orthonormal Legendre polynomials and trigonometric functions for the analysis of plane structures, has been explored in (Silva 
et al., 2004). Another important contribution, was given by (De Almeida et al. 1996), studying the bounds and error for adaptive refinement, using hybrid stress and hybrid displacement elements, for linear analysis. As mentioned before, the use of hybrid-mixed models introduces a great flexibility in the choice of the approximation functions. This fact, made it possible to use functions with special properties, that cannot be used in classical finite element formulations (Pereira et al., 1996b). Complete sets of orthonormal Legendre polynomials, are used to define the approximation bases required by the 4f-HMS model. The generation and manipulation of such functions, is fully described in (Pereira et al. 1996a; Pereira et al., 1996b; Spiegel et al., 1999). The Legendre polynomials can be defined using the Rodriguez Eq. (29).

$$
P_{n}(x)=\frac{1}{2^{n} n !} \times \frac{d^{n}}{d x^{n}}\left(x^{2}-1\right)^{n}, \text { with } \quad \lambda_{n}=\sqrt{\frac{2 n+1}{2}}
$$
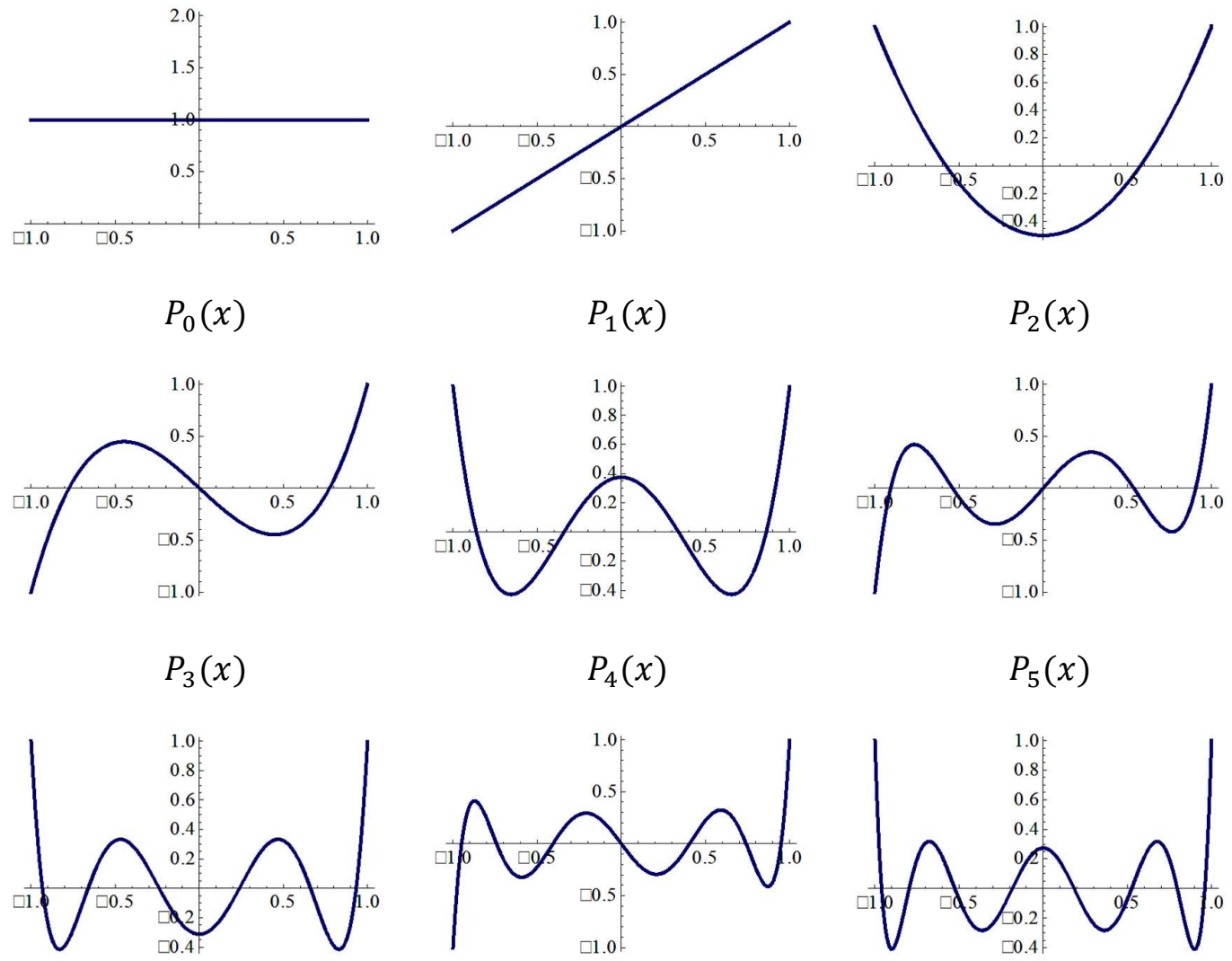

$P_{6}(x)$

$$
P_{7}(x)
$$

$P_{8}(x)$

Fig. 1. Legendre Polynomials in 1D space.

To illustrate the main difference between the Legendre approximation functions, and the approximation functions of CFE, the normalized Legendre functions are displayed for 1D case and 2D case. Fig. 1 presents the Legendre polynomials up to the $8^{\text {th }}$ degree. Tensor products, involving $1 \mathrm{D}$ bases in each coordinate direction, lead to the construction of Legendre polynomial bases for 2D domains. Fig. 2 presents the nine 2D functions defined using all 1D Legendre polynomials, up to the $2^{\text {nd }}$ degree. In the case of a 3D domain, the approximation functions are built using the $3^{\text {rd }}$ direction $z$. These approximation functions, do not obey any of the kinematic boundaries, contrary to the ones used in CFE. This is possible in $4 \mathrm{f}-\mathrm{HMS}$, since in this formulation the kinematic boundary condition is only enforced using the 
Galerkin method (as referred in equation (14)). Therefore, it is expected some discontinuity along the kinematic boundary in the deformed shape, when using Legendre polynomials with 4f-HMS.
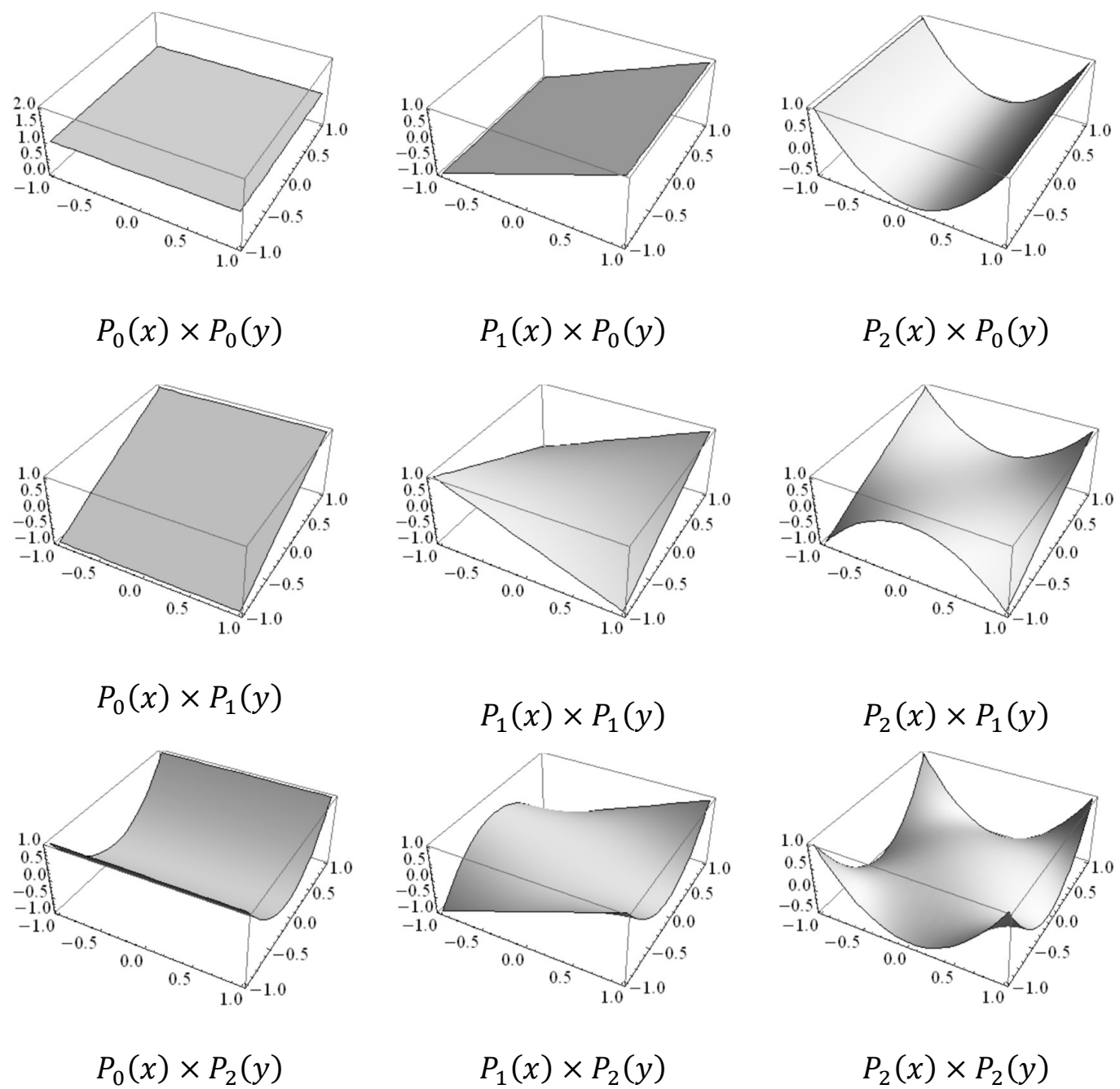

Fig. 2. - Legendre Polynomials in 2D space.

As referred before, one of the main advantages of using these polynomials, is the possibility of avoiding any numerical integration for linear analysis. It is always possible to define analytical expressions, for all structural operators, even for cases where orthogonality is not presented.

$$
\begin{aligned}
& \left\{\begin{array}{l}
\int_{-1}^{1} P_{i}(x) P_{j}(x) d x=1 \text { if } i=j \\
\int_{-1}^{1} P_{i}(x) P_{j}(x) d x=0 \text { otherwise }
\end{array}\right. \\
& \left\{\begin{array}{l}
\int_{-1}^{1} P_{i}(x) P_{j}(x) x d x=\frac{i}{2 \lambda_{i} \lambda_{j}} \text { if } i=j+1 \\
\int_{-1}^{1} P_{i}(x) P_{j}(x) x d x=\frac{i}{2 \lambda_{i} \lambda_{j}} \text { if } i=j-1 \\
\int_{-1}^{1} P_{i}(x) P_{j}(x) x d x=0 \quad \text { otherwise }
\end{array}\right.
\end{aligned}
$$




$$
\begin{aligned}
& \iint_{-1}^{1} P_{i}(x) P_{j}(x) x^{2} d x=\frac{4 i^{3}+6 i^{2}-1}{2(2 i-1)(2 i+3) \lambda_{i}^{2}} \text { if } i=j \\
& \iint_{-1}^{1} P_{i}(x) P_{j}(x) x^{2} d x=\frac{j(j-1)}{2(2 j-1) \lambda_{i} \lambda_{j}} \text { if } i=j-2 \\
& \int_{-1}^{1} P_{i}(x) P_{j}(x) x^{2} d x=\frac{i(i-1)}{2(2 i-1) \lambda_{i} \lambda_{j}} \text { if } i=j+2 \\
& \int_{-1}^{1} P_{i}(x) P_{j}(x) x^{2} d x=0 \quad \text { otherwise } \\
& \iint_{-1}^{1} P_{i}(x) P_{j}(x) x^{3} d x=\frac{j(j-1)(j-2)}{2(2 j-1)(2 j-3) \lambda_{i} \lambda_{j}} \text { if } i=j-3 \\
& \int_{-1}^{1} P_{i}(x) P_{j}(x) x^{3} d x=\frac{3 j\left(j^{2}-2\right)}{2\left(4 j^{2}-9\right) \lambda_{i} \lambda_{j}} \text { if } i=j-1 \\
& \left\{\int_{-1}^{1} P_{i}(x) P_{j}(x) x^{3} d x=\frac{3 i\left(i^{2}-2\right)}{2\left(4 i^{2}-9\right) \lambda_{i} \lambda_{j}} \text { if } i=j+1\right. \\
& \int_{-1}^{1} P_{i}(x) P_{j}(x) x^{3} d x=\frac{i(i-1)(i-2)}{2(2 i-1)(2 i-3) \lambda_{i} \lambda_{j}} \text { if } i=j+3 \\
& \int_{-1}^{1} P_{i}(x) P_{j}(x) x^{3} d x=0 \quad \text { otherwise } \\
& \begin{cases}\int_{-1}^{1} P_{i}(x) \frac{\partial P_{j}(x)}{\partial x} d x=2 \lambda_{i} \lambda_{j} & \text { if } i<j \cap i+j \text { odd } \\
\int_{-1}^{1} P_{i}(x) \frac{\partial P_{j}(x)}{\partial x} d x=0 & \text { otherwise }\end{cases} \\
& \left\{\begin{array}{l}
\int_{-1}^{1} P_{i}(x) \frac{\partial P_{j}(x)}{\partial x} x d x=i \quad \text { if } i=j \\
\int_{-1}^{1} P_{i}(x) \frac{\partial P_{j}(x)}{\partial x} x d x=2 \lambda_{i} \lambda_{j} \quad \text { if } i<j \cap i+j \text { even } \\
\int_{-1}^{1} P_{i}(x) \frac{\partial P_{j}(x)}{\partial x} x d x=0 \quad \text { otherwise }
\end{array}\right. \\
& \iint_{-1}^{1} P_{i}(x) \frac{\partial P_{j}(x)}{\partial x} x^{2} d x=\frac{i(i-1)}{2 \lambda_{i} \lambda_{j}} \quad \text { if } i=j+1 \\
& \left\{\int_{-1}^{1} P_{i}(x) \frac{\partial P_{j}(x)}{\partial x} x^{2} d x=\frac{3 j^{2}-j-1}{2 \lambda_{i} \lambda_{j}} \quad \text { if } i=j-1\right. \\
& \int_{-1}^{1} P_{i}(x) \frac{\partial P_{j}(x)}{\partial x} x^{2} d x=2 \lambda_{i} \lambda_{j} \quad \text { if } i<j \cap i+j \text { even } \\
& \int_{-1}^{1} P_{i}(x) \frac{\partial P_{j}(x)}{\partial x} x^{2} d x=0 \quad \text { otherwise }
\end{aligned}
$$




$$
\begin{aligned}
& \left\{\begin{array}{l}
\int_{-1}^{1} \frac{\partial P_{i}(x)}{\partial x} \frac{\partial P_{j}(x)}{\partial x} d x=\left(i^{2}+i\right) \lambda_{i} \lambda_{j} \quad \text { if } i \leq j, i>0, j>0, i+j \text { even } \\
\int_{-1}^{1} \frac{\partial P_{i}(x)}{\partial x} \frac{\partial P_{j}(x)}{\partial x} d x=\left(j^{2}+j\right) \lambda_{i} \lambda_{j} \quad \text { if } i>j, i>0, j>0, i+j \text { odd } \\
\int_{-1}^{1} \frac{\partial P_{i}(x)}{\partial x} \frac{\partial P_{j}(x)}{\partial x} d x=0 \quad \text { otherwise }
\end{array}\right. \\
& \left\{\begin{array}{l}
\int_{-1}^{1} \frac{\partial P_{i}(x)}{\partial x} \frac{\partial P_{j}(x)}{\partial x} x d x=\left(i^{2}+i\right) \lambda_{i} \lambda_{j} \quad \text { if } i<j, i>0, j>0, i+j \text { odd } \\
\int_{-1}^{1} \frac{\partial P_{i}(x)}{\partial x} \frac{\partial P_{j}(x)}{\partial x} x d x=\left(j^{2}+j\right) \lambda_{i} \lambda_{j} \quad \text { if } i>j, i>0, j>0, i+j \text { odd } \\
\int_{-1}^{1} \frac{\partial P_{i}(x)}{\partial x} \frac{\partial P_{j}(x)}{\partial x} x d x=0 \quad \text { otherwise }
\end{array}\right.
\end{aligned}
$$

It is still possible to integrate numerically, some non-common examples:

$$
\begin{aligned}
& \left\{\begin{array}{l}
\int_{-1}^{1} P_{i}(x) d x=\sqrt{2} \quad \text { if } i=0 \\
\int_{-1}^{1} P_{i}(x) d x=0 \quad \text { otherwise }
\end{array}\right. \\
& \int_{-1}^{1} \int_{-1}^{1} P_{0}(x) P_{0}(y) d x d y=2 \\
& \int_{-1}^{1} \int_{-1}^{1} P_{1}(x) P_{0}(y) x d x d y=\frac{2}{\sqrt{3}}
\end{aligned}
$$

\section{Numerical Applications}

In this section, some numerical examples in which the analytical solution is known, are tested for isotropic elastic models. The first test consists of a rectangular plate with a crack in the middle, and subjected to tensile stress in the top. This example possesses an almost exact solution, presented in the numerical work of (Freitas et al., 1999). In the second test, is the classical L-Shape Plate under tension, in which an analytical solution is provided by (Shephard et al., 1989). Finally a short cantilever presented in the work of (Pereira, 1996), which was also tested with equilibrium finite element formulation, is assessed and compared with the new proposed finite element. In all examples the 4f-HMS is directly compared with CFE.

\section{Cracked Rectangular Plate}

The rectangular plate, with a central crack shown in Fig. 3 is a typical example, where the use of a higher-order scheme is recommended, due to the existence of a strong singularity at the tip of the crack. Owing to symmetry conditions, only $1 / 4$ of the plate is analysed. Fig. 4 shows the different meshes used in the analysis, with the rectangular elements taken into consideration. The mesh with non-rectangular elements is used, to illustrate the effect of mesh distortion both in the governing system sparsity and in 
the accuracy of the solution. The $p$ - refinement procedure, involves the use of a given finite element mesh with increasing values for degree of the Legendre polynomial. It is assumed here that degree parameter ranges from values given in Table 1. For the CFE, a regular structured mesh was used. Combining the different finite (rectangular) element meshes, with the different degrees of approximation, it is possible to define a set of 50 different discretizations, used to illustrate the efficiency of $p$-adaptive procedures. A small test is also presented for the $h$-refinement, in which the degree of the Legendre polynomial is fixed at 2 , for the all the meshes.

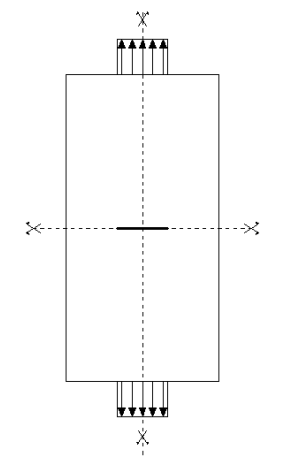

a)

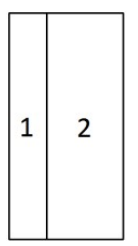

2 Elements
Fig. 3. Plane cracked structure a) Rea

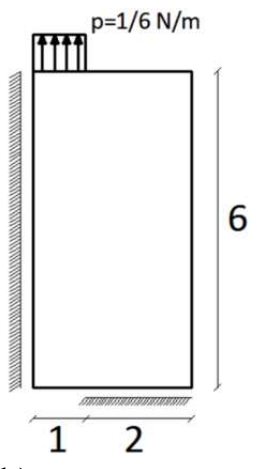

b)

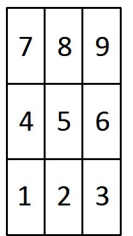

9 Elements

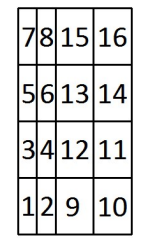

16 Elements

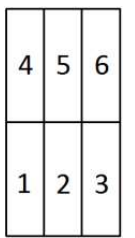

6 Elements

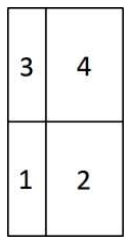

4 Elements

Fig. 4. - Adopted mesh for the plane cracked structure.

Table 1. Adopted discretizations for the plane cracked structure.

\begin{tabular}{clcccccccccc}
\hline & \multicolumn{1}{c}{ Discretizations } \\
\hline \multirow{2}{*}{ 2 Elements } & Legendre Degree & 2 & 3 & 4 & 5 & 9 & 13 & 15 & 17 & 20 & 50 \\
& Degrees of Freedom & 148 & 264 & 412 & 592 & 1632 & 3184 & 4152 & 5248 & 7132 & 41812 \\
\hline \multirow{2}{*}{4 Elements } & Legendre Degree & 2 & 3 & 4 & 5 & 9 & 11 & 13 & 15 & 17 & 40 \\
& Degrees of Freedom & 290 & 519 & 812 & 1169 & 3237 & 4655 & 6329 & 8259 & 10445 & 53984 \\
\hline \multirow{2}{*}{6 Elements } & Legendre Degree & 2 & 3 & 4 & 5 & 7 & 9 & 11 & 15 & 20 & 30 \\
& Degrees of Freedom & 432 & 774 & 1212 & 1746 & 3102 & 4842 & 6966 & 12366 & 21276 & 46296 \\
\hline \multirow{2}{*}{ 9 Elements } & Legendre Degree & 2 & 3 & 4 & 5 & 7 & 9 & 11 & 15 & 17 & 20 \\
& Degrees of Freedom & 644 & 1155 & 1810 & 2609 & 4639 & 7245 & 10427 & 18519 & 23429 & 48829 \\
\hline \multirow{2}{*}{16 Elements } & Legendre Degree & 2 & 3 & 4 & 5 & 7 & 9 & 11 & 15 & 17 & 20 \\
& Degrees of Freedom & 1140 & 2046 & 3208 & 4626 & 8230 & 12858 & 18510 & 32886 & 41610 & 56616 \\
\hline \multirow{2}{*}{ CFE } & Degrees of Freedom & 182 & 462 & 992 & 3782 & 14762 & & & & &
\end{tabular}

For the isotropic model, using the following mechanical properties $E=1.0 \mathrm{~Pa}, v=0.3$, thickness $=$ 1.0, an almost "exact" elastic strain energy can be admitted as $U=0.0458402901388889 \mathrm{~J}$. This numerical value is given in the work of Freitas et al. (1999), and is obtained by applying a dual extrapolation technique, to two series of kinematically and statically admissible solutions, which provide lower and upper bounds for the exact solution. In Fig. 5 it is presented the strain energy convergence a) rate and b) absolute error Eq. (42), for all regular meshes presented in Fig. 4, using all the discretizations 
adopted in Table 1. In the same graphic, the elastic strain energy is also presented for the CFE, using Q4 elements with full numerical integration scheme. An absolute error (in this case a positive value) is used, to allow a logarithmic scale.

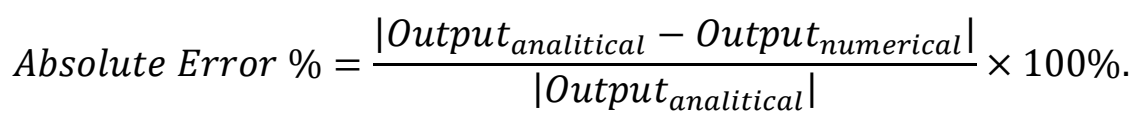

For this numerical test, all meshes except one presented a perfectly monotonic convergence (excluding the mesh with 2 finite elements). All of these, also presented a pure linear convergence when a logarithmic scale is used (when plotting the absolute error). This convergence behaviour is expected in hybrid stress models, but in hybrid mixed stress models some inconsistences are also expected. This isn't any different in the new hybrid double mixed stress (4f-HMS) model, in which the 2 element convergence presents a non-monotonic convergence for lower degrees of approximation. Contrary to CFE, in which the convergence is monotonic (due to its displacement formulation), in the $4 \mathrm{f}-\mathrm{HMS}$ there is no assurance on its evolution. Also analysing Fig. 5 b), it is possible to conclude that for smaller degrees of freedom (bellow 1000), even with only 2 elements the 4f-HMS presents more accurate results than the CFE.

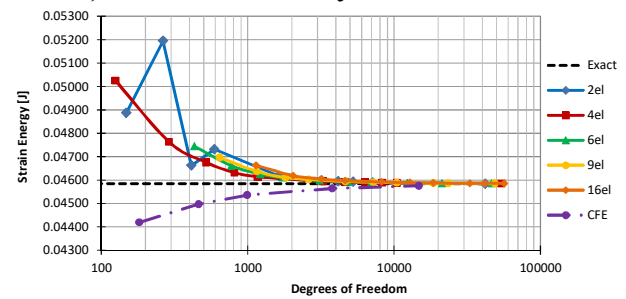

a)

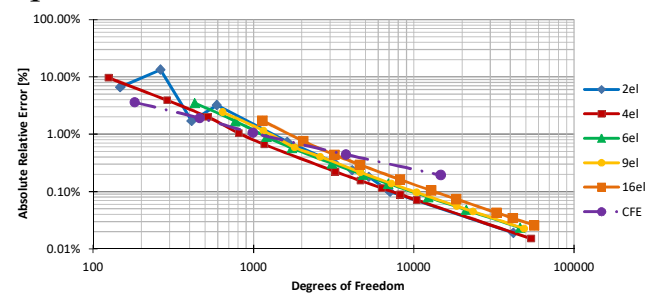

b)

Fig. 5. - Energetic convergence for the cracked rectangular plate, a) Strain energy vs DOF, b) Absolute error of the strain energy vs DOF.

Fig. 6 displays the strain energy convergence ( $a$ - rate and $b$ - absolute error) for the 4 element mesh, with regular and distorted case (Fig. 4), using all the discretizations adopted in Table 1. In this case, the 4f-HMS presents an even worst monotonic convergence, which presents a lower bound for the distorted mesh. It can also be pointed out that, although, the level of distortion is high, the rate of convergence of the $4 \mathrm{f}-\mathrm{HMS}$ is also high.

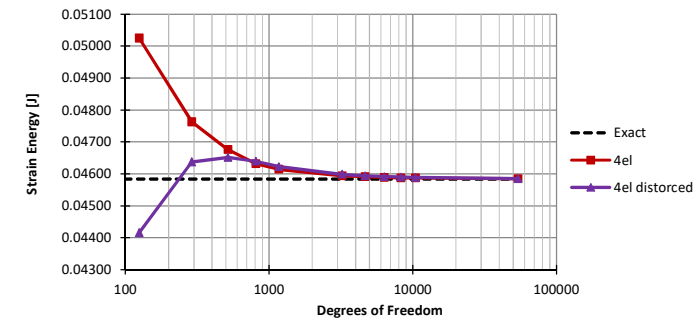

a)

Fig. 6. Energetic convergence for the cracked rectangular plate, only with 4 elements using straight and distorted meshes, a) Strain energy vs DOF, b) Absolute error of the strain energy vs DOF.

The evolution of the vertical displacement and stress at the top left node of Fig. 3 b), is displayed in Fig. 7. The purpose of this test is to assess the monotonic convergence of the $4 \mathrm{f}-\mathrm{HMS}$, for the displacement and stress field. The theoretical value of the vertical stress, is $1 / 6 \mathrm{~N} / \mathrm{m}^{2}$, but the exact value of the vertical displacement is unknown. 


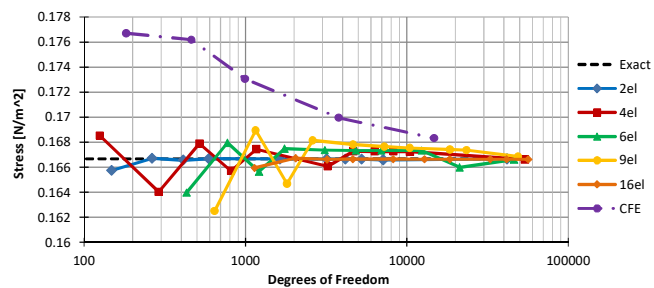

a)

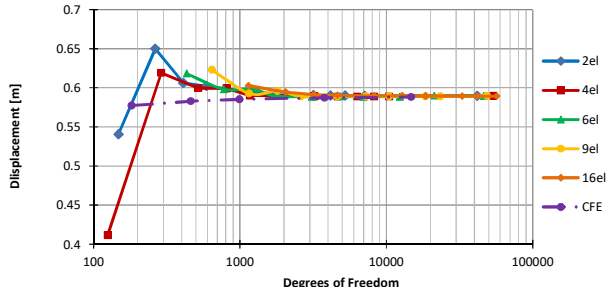

b)

Fig. 7. Convergence for the cracked rectangular plate at the top left node, a) Vertical stress vs DOF, b) Vertical displacement vs DOF.

It is clear in Fig. 7 a), that there is no monotonic convergence in all discretizations with the 4f-HMS, contrary to the one observed in CFE. Also, the rate of convergence in the 4f-HMS is clearly superior than the CFE in terms of stress behaviour, for regular meshes. This is expected, since in the 4f-HMS the stress field is directly approximated. On the other hand, this conclusion would be erroneous in Fig. 7 b). In fact, for some discretizations, the CFE presents almost the same rate convergence as the 4f-HMS for the displacement field. Once again, the displacements with the 4f-HMS are not always monotonic, contrary to the CFE which are always lower bound due to its stiffer formulation.

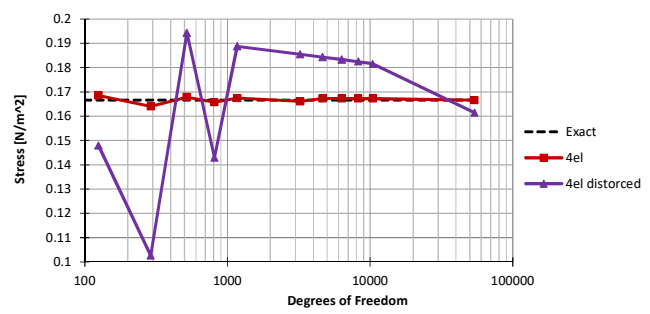

a)

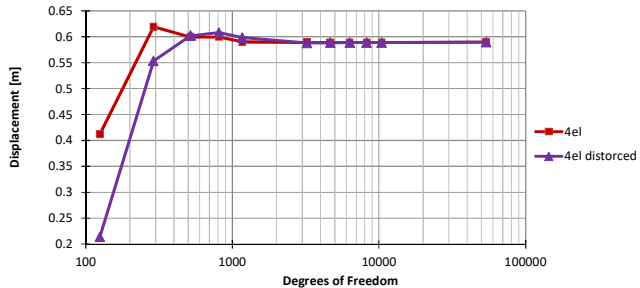

b)

Fig. 8. Convergence for the cracked rectangular plate, only with 4 elements using straight and distorted meshes, a) Vertical stress vs DOF, b) Vertical displacement vs DOF.

In Fig. 8, the convergence rate of a fully regular and a totally distorted mesh, with 4f-HMS are compared, with the purpose of studying the Gibbs effect in the static and kinematic boundary. In Fig. 8 a), the distorted mesh presents some difficulties converging in to a stress level of $1 / 6 \mathrm{~N} / \mathrm{m}^{2}$, even for the number of degrees of freedom of more than 40000 . This is due to the Gibbs effect presented in the approximation of the stress field by Legendre polynomials. This effect is enchased when discontinuities occur in the static boundary, when after $x=1.0$ the stress level is exactly $0.0 \mathrm{~N} / \mathrm{m}^{2}$, as shown in Fig. 9. In any case this problem is more visible in distorted meshes with 4f-HMS, but for regular meshes this problem is less noticeable. This can be concluded by observing Fig. 8 a), where for 2 and 16 regular elements the value is almost stable around $1 / 6 \mathrm{~N} / \mathrm{m}^{2}$. Since the kinematic boundary of the impose problem presents no discontinuity, it is expected a good convergence rate for the displacement field of the regular and the distorted mesh, which can be concluded by observing Fig. 8 b). Although the 4 element mesh is heavily distorted, the convergence rate of the displacement is similar to the 4 element regular mesh.

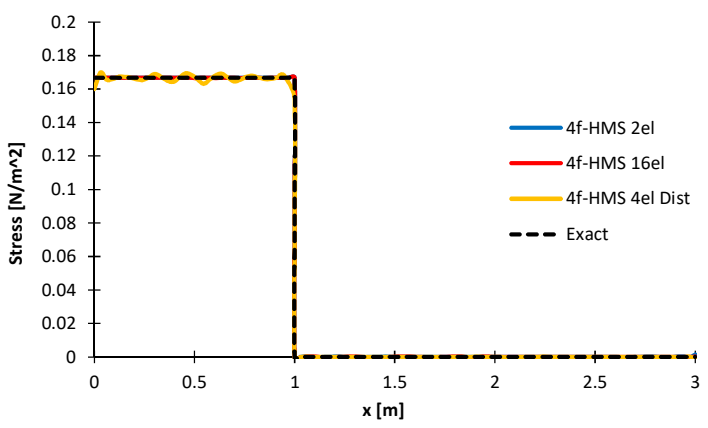

Fig. 9 Vertical stress distribution in the top static boundary of the cracked rectangular plate 
In Fig. 10, the evolution of the shear stress field $\tau_{x y}$ for the $p$ - refinement is displayed for the 4 elements mesh. Although the convergence is extremely fast, the use of Legendre polynomials presents some instabilities due to the Gibbs effect, as it can be seen in the $40^{\text {th }}$ degree approximation. This drawback, in the use of Legendre polynomials for the approximation functions, is also observed in the work of (Mendes et al. 2009). The $h$ - refinement is also presented in Fig. 11, maintaining the degree of approximation 2 for the Legendre polynomial and using the same stress scale as in Fig. 10. Due to the low level of finite elements and degree approximation used, the rate convergence is very low. To present the effectiveness of the 4f-HMS with distorted elements, a p- refinement is displayed in Fig. 12, using the same stress scale as in Fig. 10. Even with high distortion, the convergence rate is extremely fast with low DOF, but in this case the Gibbs effect is much more pronounced.

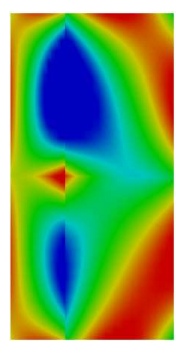

$2^{\text {nd }}$ degree

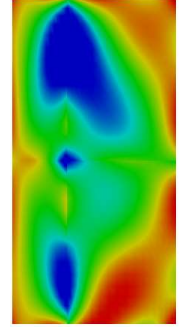

$3^{\text {rd }}$ degree

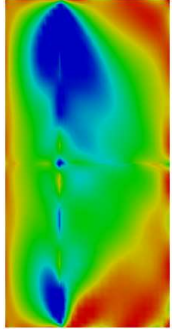

$5^{\text {th }}$ degree

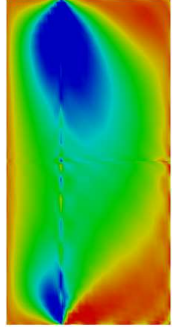

$9^{\text {th }}$ degree

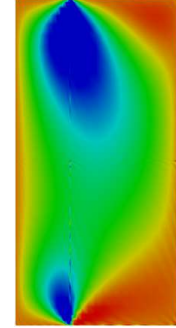

$17^{\text {th }}$ degree

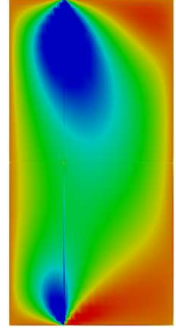

$40^{\text {th }}$

degree

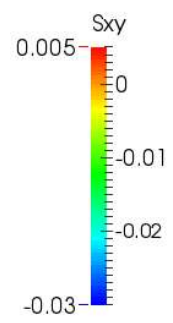

Fig. 10. Evolution of the shear stress distribution, for the 4 elements mesh, using $p$ - refinement.

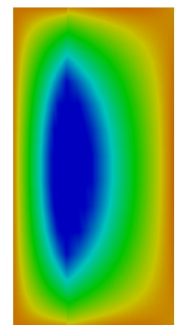

2

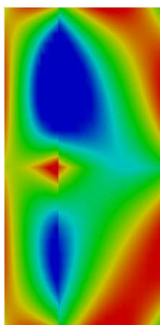

4

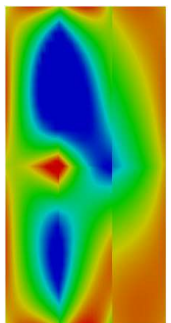

6

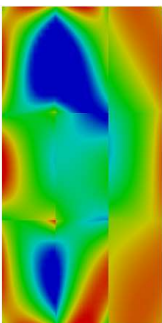

9

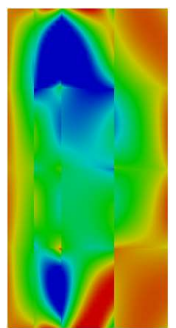

16

\section{Elements Elements Elements Elements Elements}

Fig. 11. Evolution of the shear stress distribution, for the 4 elements mesh, using $h$ - refinement.

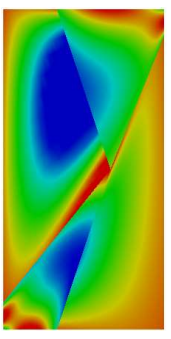

$2^{\text {nd }}$ degree

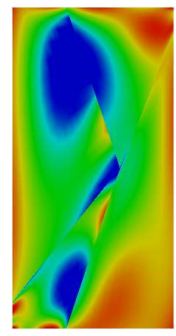

$3^{\text {rd }}$ degree

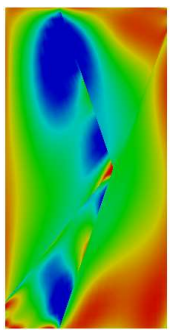

$4^{\text {th }}$ degree

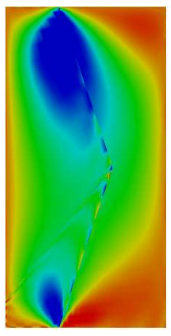

$9^{\text {th }}$ degree

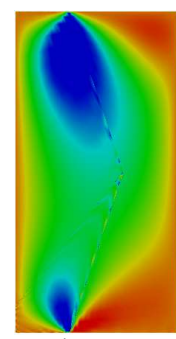

$13^{\text {th }}$

degree

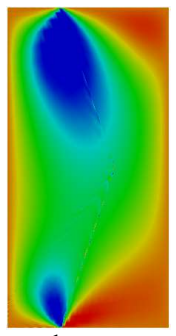

$17^{\text {th }}$

degree

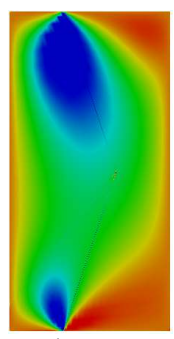

$40^{\text {th }}$

degree

Fig. 12. Evolution of the shear stress distribution, for the 4 elements distorted mesh, using $p$ refinement

\section{L-Shaped Plate Under Tension}

The L-Shaped plate shown in Fig. 13 is another typical example, where the use of a higher-order scheme is recommended, due to the existence of a strong singularity in the interior corner. This problem is particularly important, since it is known for showing some numerical instabilities due to the Gibbs effect alongside the static boundary. Owing to symmetry conditions, only $1 / 4$ of the plate is analysed. 
Fig. 14 shows the different meshes used in the analysis, with the rectangular elements considered. It is assumed here, that the degree parameter of the Legendre polynomial approximation, ranges from values given in Table 2. Combining the different finite (rectangular) element meshes, with the different degrees of approximation, it is possible to define a set of 33 different discretizations, used to illustrate the efficiency of $p$ - adaptive procedures.

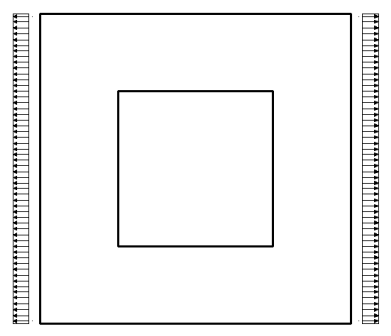

a)

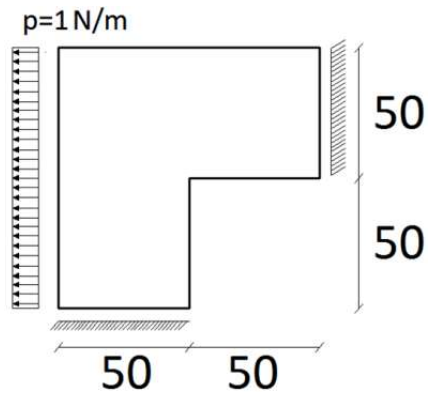

b)

Fig. 13. Plane structure a) Real case, b) Analysed structure.

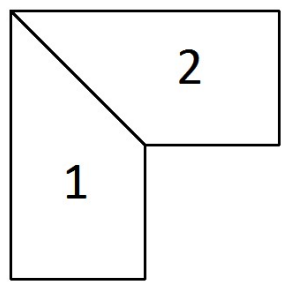

2 Elements

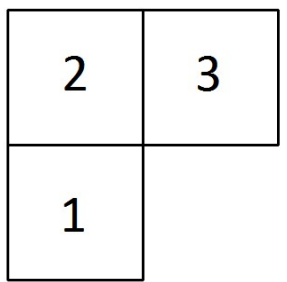

3 Elements

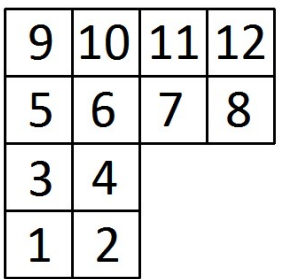

12 Elements

Fig. 14. Adopted mesh for the L-shaped plane.

Table 2. Adopted discretizations for the L-shaped plane

\begin{tabular}{|c|c|c|c|c|c|c|c|c|c|c|c|c|}
\hline \multicolumn{13}{|c|}{ Discretizations } \\
\hline \multirow{2}{*}{2 Elements } & Legendre Degree & 1 & 2 & 3 & 4 & 5 & 9 & 13 & 15 & 17 & 20 & 50 \\
\hline & Degrees of Freedom & 64 & 148 & 264 & 412 & 592 & 1632 & 3184 & 4152 & 5248 & 7132 & 41812 \\
\hline \multirow{2}{*}{3 Elements } & Legendre Degree & 1 & 2 & 3 & 4 & 5 & 9 & 11 & 13 & 15 & 17 & 40 \\
\hline & Degrees of Freedom & 96 & 222 & 396 & 618 & 888 & 2448 & 3516 & 4776 & 6228 & 7872 & 40578 \\
\hline \multirow{2}{*}{12 Elements } & Legendre Degree & 1 & 2 & 3 & 4 & 5 & 7 & 8 & 9 & 11 & 15 & 20 \\
\hline & Degrees of Freedom & 372 & 864 & 1548 & 2424 & 3492 & 6204 & 7848 & 9684 & 13932 & 24732 & 92592 \\
\hline CFE & Degrees of Freedom & 192 & 352 & 682 & 1472 & 2562 & 9922 & 60802 & & & & \\
\hline
\end{tabular}

For the isotropic model, using the following mechanical properties $E=10^{5} \mathrm{~Pa}, v=0.3$, thickness $=$ 1.0, an exact elastic strain energy can be admitted as $U=0.1555(6) \mathrm{J}$. This analytical value is given in the work of Shephard et al. (Shephard et al. 1989). In Fig. 15 it is presented the strain energy convergence a) rate and b) absolute error, for all meshes presented in Fig. 14, using all the discretizations adopted in Table 2. For the CFE, a regular structured mesh was used. The main difference from the previous example is that, even for regular meshes some lower bounds may be found, meaning once again the 4f-HMS presents no monotonic consistence, in terms of strain energy convergence. Another difference, is when a logarithmic scale is used to plot the absolute error, the variation is no longer pure linear. This is due to the linear load along the static boundary, and the lower level of singularity. 


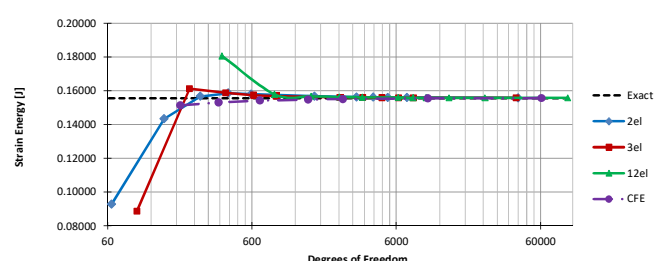

a)

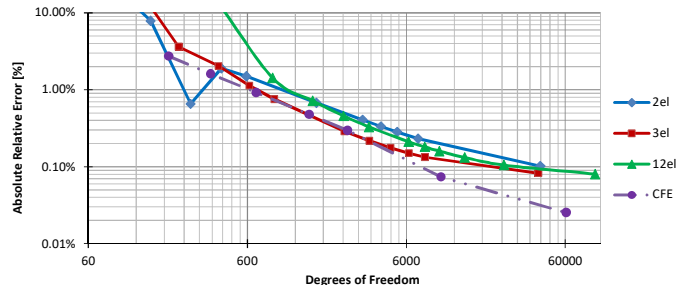

b)

Fig. 15. Energetic convergence for the L-shaped plate, a) Strain energy vs DOF, b) Absolute error of the strain energy vs DOF

In Fig. 16, the evolution of static and kinematic boundaries values is presented for both 4f-HMS and CFE. A peculiar non-convergence appears in Fig. 16 a) for the mesh with 2 elements (which are distorted) even for more than 40000 number of degrees of freedom. Once again, this is a problem derived from the approximation of Legendre polynomials in the 4f-HMS stress field. Observing Fig. 17, it is possible to conclude that in the top and bottom of the static boundary, the noise due to the Gibbs effect is extremely large, leading to heavy jumps near the borders. Although this is only visible for the 2 element mesh, the meshes with 3 and 16 elements also present some convergence issues in the static boundary, as can be observed in Fig. 18 in which this problem is atoned, but present. It is also important to point out that, for all $4 \mathrm{f}-\mathrm{HMS}$ discretizations, the resultant stress (force) is always equal to $200 \mathrm{~N}$.

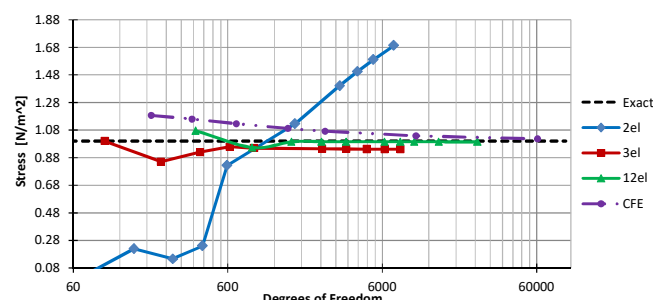

a)

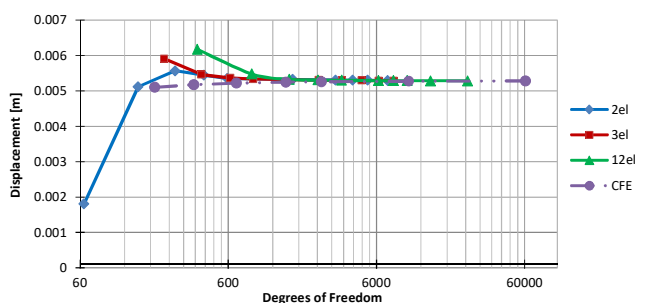

b)

Fig. 16. Convergence for the L-shaped plate in the bottom left node, a) Horizontal stress vs DOF, b) Horizontal displacement vs DOF

In Fig. $16 \mathrm{~b}$ ), the convergence rate of the 4f-HMS is compared with the CFE for the kinematic boundary, for the bottom left node. Due to the rate inefficiency at the stress level, some convergence issues occur for the displacement with the 4f-HMS using low degrees of freedom, when directly compared with CFE.

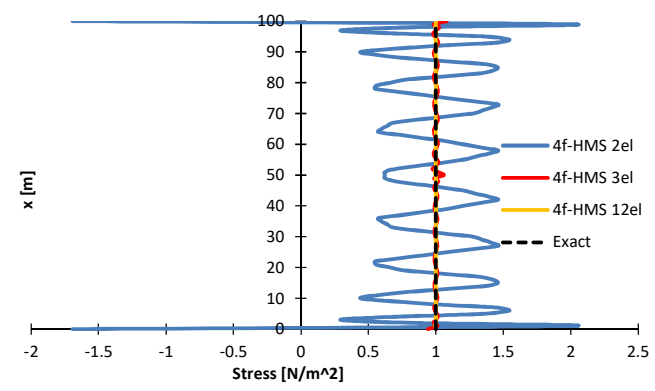

Fig. 17. Horizontal stress distribution in the left static boundary of the L-shaped plate, for the higher discretizations

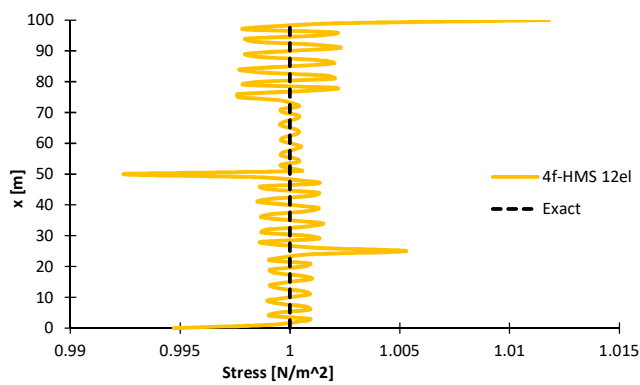

Fig. 18. Zoomed horizontal stress distribution in the left static boundary of the L-shaped plate, only for 12 element model

In Fig. 19, the evolution of the shear stress field $\sigma_{x y}$ for the $p$-refinement is displayed for the 2 elements mesh, using a deformed shape at the scale of 5000. Although the convergence is extremely fast, the use of Legendre polynomials presents some instabilities due to the Gibbs effect, as in the previous 
example. This is more perceptible along the diagonal kinematic boundary between elements. For the $1^{\text {st }}$ discretization, since the degree of approximation of the stress field is only 2 (and consequently for the displacement field 1), it is expected a severe discontinuity along the kinematic boundary. This is also due to the fact that in the 4f-HMS formulation both Neumann and Dirichlet conditions are only enforced using the weighted residual method (Galerkin method). Even in the $2^{\text {nd }}$ and $3^{\text {rd }}$ discretization, a smaller kinematic boundary error is observed.

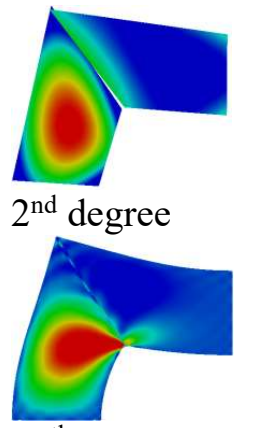

$13^{\text {th }}$ degree

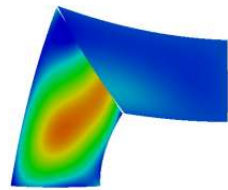

$3^{\text {rd }}$ degree

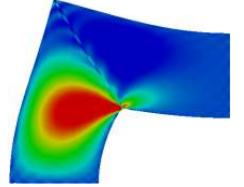

$15^{\text {th }}$ degree
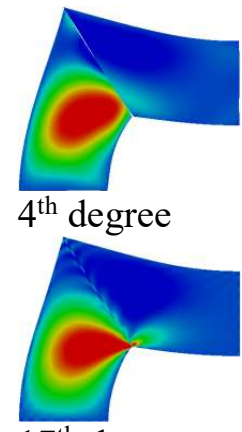

$17^{\text {th }}$ degree

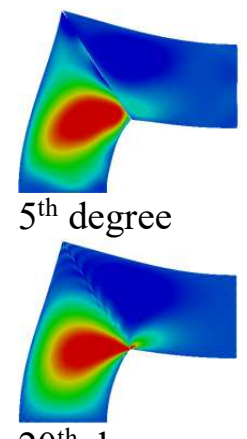

$20^{\text {th }}$ degree

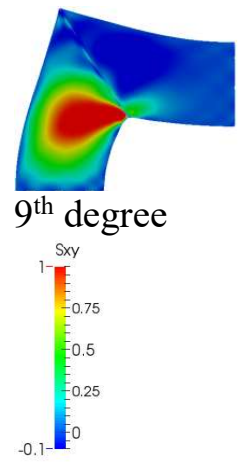

Fig. 19. Evolution of the shear stress distribution, for the 2 elements mesh, using p- refinement

Contrary to the previous example, the Gibbs effect has almost completely vanished, as can been seen in Fig. 20 for the best discretization for each mesh presented in Table 2.

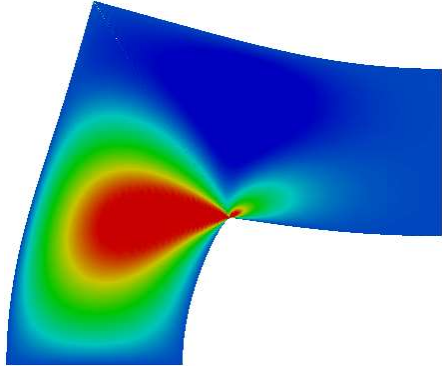

2 Elements

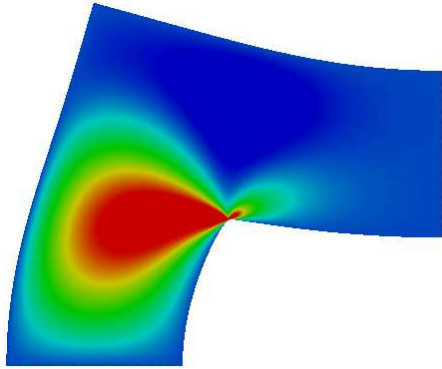

3 Elements

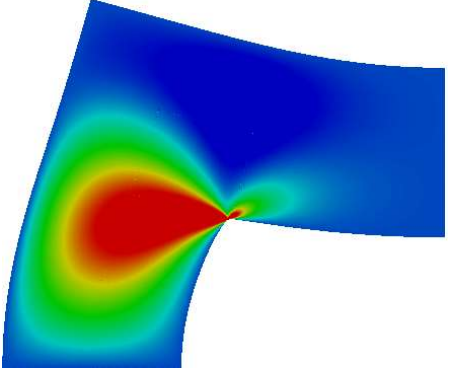

12 Elements

Fig. 20. Shear stress distribution for the 3 meshes using the best discretization

\section{Short Cantilever}

In the following example, the short cantilever depicted in Fig. 21, in which the vertical displacement is generally governed by shear flexibility, is tested using 4f-HMS finite element model. This benchmark example is usually studied by other researchers (Pereira, 1996), when testing new finite element formulations in terms of strain energy convergence. This particular example was performed by (Pereira, 1996), using equilibrium finite element, more specifically hybrid stress (HS) formulation (De Almeida et al., 1991). For the isotropic model, an almost "exact" elastic strain energy can be admitted as $U=$ 0.100361948J. This numerical value is given in the work (Pereira 1996), and is obtained by applying a dual extrapolation technique to two series of kinematically and statically admissible solutions, which provide lower and upper bounds for the exact solution. In Table 3, the adopted discretizations for the $4 \mathrm{f}-$ HMS, CFE and HS models are displayed, including the degree of the Legendre polynomial approximation for the $4 \mathrm{f}-\mathrm{HMS}$ case. In 3 cases, the total number of degrees of freedom is presented. For the 4f-HMS, it was used only one distorted element, since in the previous tests this was the one that presented lower convergence rate. 


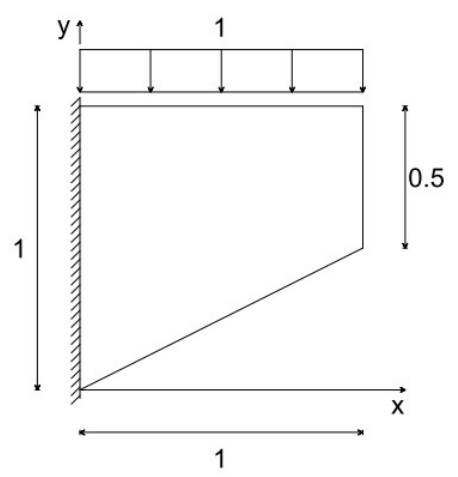

Fig. 21. Short cantilever subjected to vertical static distributed load (Pereira, 1996)

Table 3. Adopted discretizations for the short cantilever

\begin{tabular}{|c|c|c|c|c|c|c|c|c|c|c|c|c|}
\hline \multicolumn{13}{|c|}{ Discretizations } \\
\hline 4f-HMS & Legendre Degree & 1 & 2 & 3 & 4 & 5 & 9 & 13 & 15 & 17 & 20 & 50 \\
\hline 41-ПIVIS & Degrees of Freedom & 64 & 148 & 264 & 412 & 592 & 1632 & 3184 & 4152 & 5248 & 7132 & 41812 \\
\hline CFE & Degrees of Freedom & 192 & 352 & 682 & 1472 & 2562 & 9922 & 60802 & & & & \\
\hline HS & Degrees of Freedom & 74.5 & 272 & 668 & 2382 & 4406 & & & & & & \\
\hline
\end{tabular}

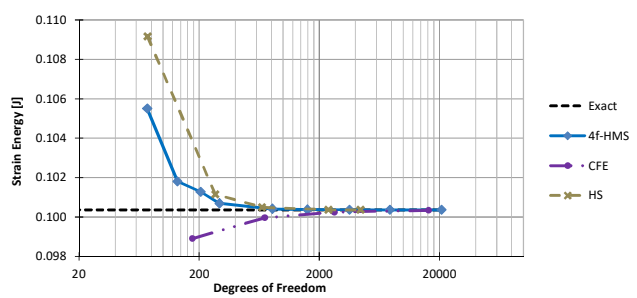

a)

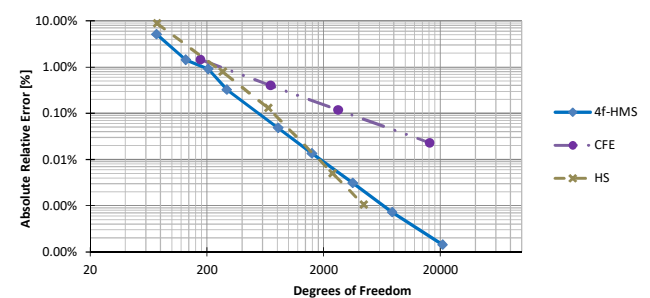

b)

Fig. 22. Energetic convergence for the short cantilever, a) Strain energy vs DOF, b) Absolute error of the strain energy vs DOF

It is displayed in Fig. 22 the strain energy a) evolution and b) absolute error, using all the discretizations adopted in Table 3. In this case, the 4f-HMS presents a poor error evolution when directly compared with HS for higher DOF. Even with the level of distortion, the rate of convergence of the 4fHMS is high when directly compared with CFE. It can be concluded that for lower level of distortion, the $4 \mathrm{f}-\mathrm{HMS}$ presents a good rate of convergence in terms of strain energy, even though it may present some instability in the static boundary as viewed before. In Fig. 23, the shear stress distribution in the deformed shape is present for several discretizations with the $4 \mathrm{f}-\mathrm{HMS}$ finite element model. This stress distribution is important since, in previous studies by other authors, it was demonstrated that it is difficult to obtain an exact result near the upper left node, mainly in the left kinematic boundary (Freitas, 1999). In terms of stress distribution, only for degrees above the $17^{\text {th }}$ it is possible to obtain a coherent shear stress distribution near the upper left node. A correct shear distribution in the left kinematic boundary is also obtained only for the $50^{\text {th }}$ degree. This is a direct consequence of the distortion of the adopted mesh with 4f-HMS. The deformed shape, presented in Fig. 23 for the first discretizations, violates the kinematic left boundary of the fixed support. Only after the $5^{\text {th }}$ degree, no visual kinematic violations are present (even though they always exist due to the 4f-HMS formulation). This effect is strongly noticeable in discretization of the $2^{\text {nd }}$ degree, since the displacement field presents a $1^{\text {st }}$ degree approximation. 

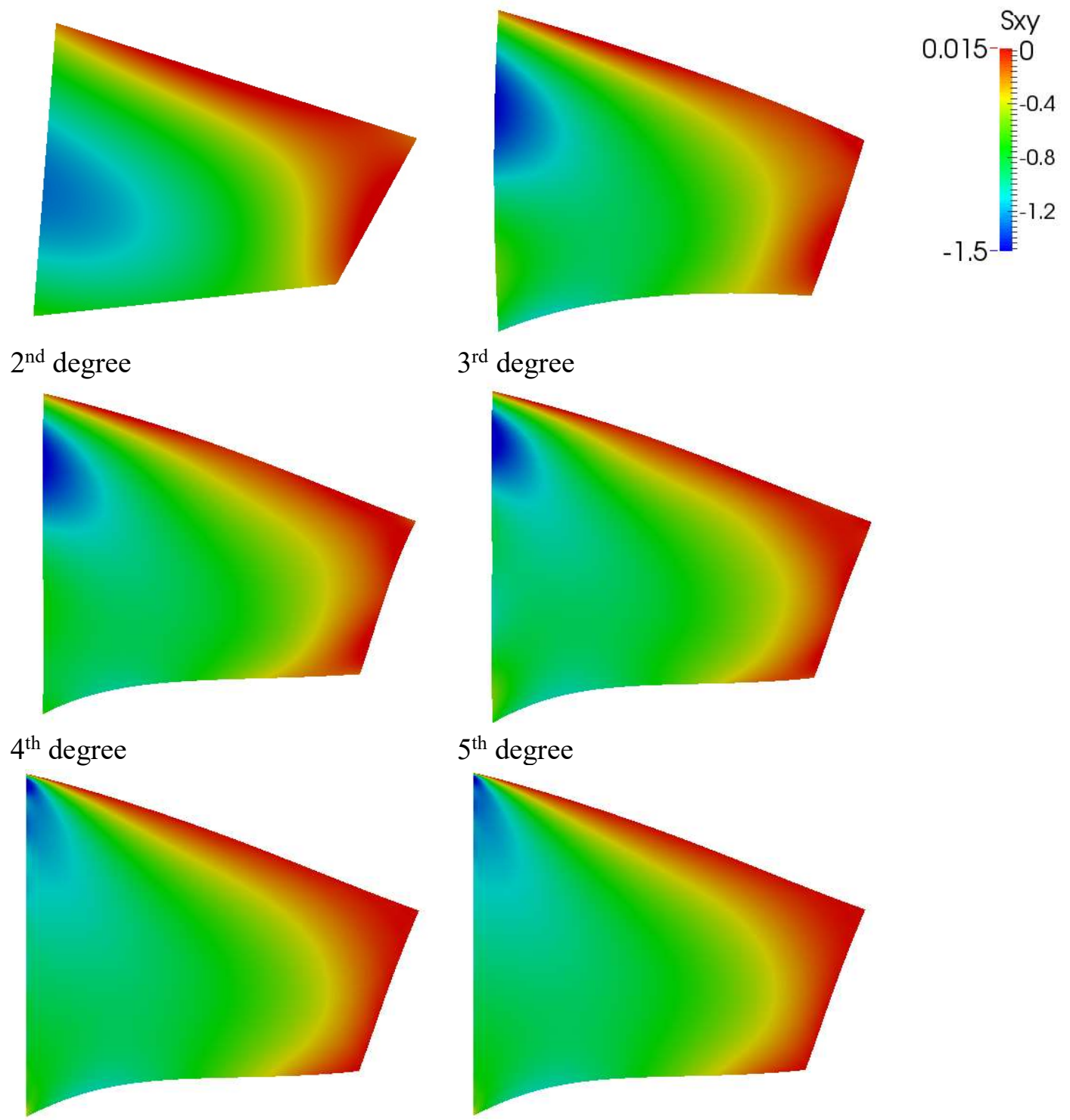

$5^{\text {th }}$ degree
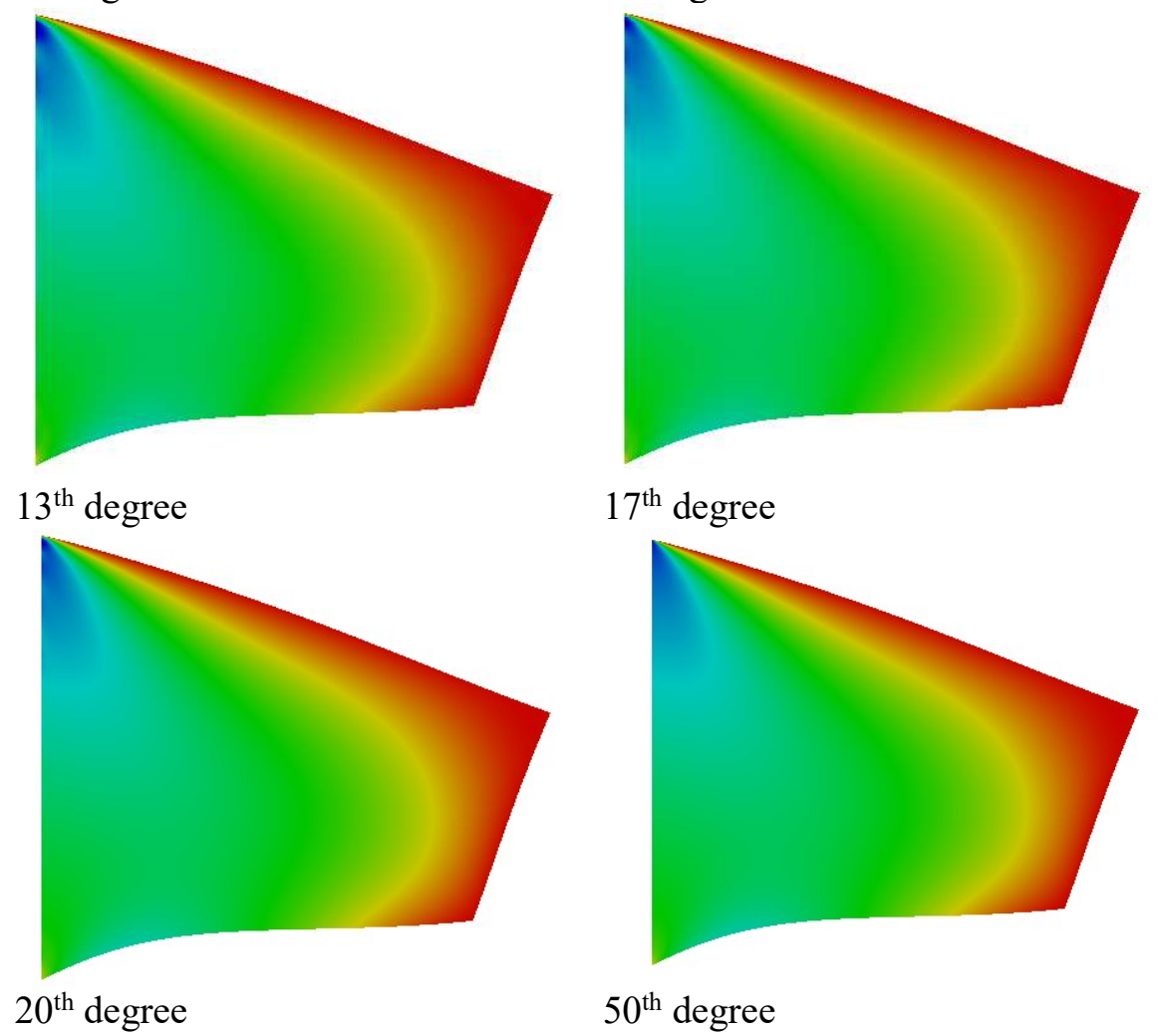

$20^{\text {th }}$ degree

$50^{\text {th }}$ degree

Fig. 23. Evolution of the shear stress distribution, using p- refinement for the short cantilever, using the stress and strain degree approximation 


\section{Conclusions}

The objective of this work consisted in the development and test of a hybrid double mixed stress finite element model for the static analysis plane elements.

- The examples presented show clearly that the model is stable, robust and leads to accurate results. Effective $p$ - and $h$ - refinement procedures can also be easily implemented, although the $h$ refinement isn't as effective as in CFE in terms of computational cost.

- The possibility of hierarchy refinement without changing the mesh, allows the use of macroelements of large dimensions, which greatly simplifies the operations of post-processing.

- Since 4f-HMS model does not verify neither equilibrium nor compatibility conditions, nothing can be concluded for the upper or lower bound of the strain energy calculated, for isotropic elastic models.

- Just like in previous studies (Mendes et al. 2009), when using hybrid mixed formulation with Legendre polynomials approximation, the Gibbs effect may cause some convergence issues in the stress field, particularly surrounding the static boundary of the imposed problem.

As future work, the first step will correspond to the verification of the LBB inf-sup condition (Babuška et al. 1997), using the 4f-HMS for static analysis. It is expected also the generalization of the models discussed here to make possible the static and dynamic analysis of physically and geometrically nonlinear structures, using mixed time integration.

\section{Acknowledgments}

The first author wishes to thank the financial support of FCT through scholarship SFRH/BPD/99902/2014.

\section{References}

Arruda, M. R.(2011) "Static and Dynamic Analysis of Concrete Structures Using Damage Mechanics". Lisbon, Instituto Superior Técnico. Ph.D. Thesis

Arruda, M. R., \& Castro, L. M. S. S.(2011a). Hybrid mixed formulation in continuum damage mechanics. CFRAC. Barcelona

Arruda, M. R., \& Castro, L. M. S. S.(2011b). Structural dynamic analysis using hybrid and mixed finite element models. Finite Elements in Analysis and Design, 57, 43-57.

Arruda, M. R. T., \& Castro, L. M. S. (2013a). A new hybrid-mixed stress model for the analysis of concrete structures using damage mechanics. Computers \& Structures, 125, 23-44.

Arruda, M. R., \& Castro, L. M. S. (2013). Static and dynamic physically non-linear analysis of concrete structures using a hybrid mixed finite element model. Advances in Engineering Software, 65, 112131.

Babuška, I., \& Narasimhan, R. (1997). The Babuška-Brezzi condition and the patch test: an example. Computer methods in applied mechanics and engineering, 140(1-2), 183-199.

Bathe, K. J.(1996). Finite Element Procedures In Engineering Analysis. Prentice-Hall Englewood Cliffs.

Castro, L. M. S. (2010). Polynomial wavelets in hybrid-mixed stress finite element models. International journal for numerical methods in biomedical engineering, 26(10), 1293-1312.

Castro, L. S., \& Barbosa, A. R. (2006). Implementation of an hybrid-mixed stress model based on the use of wavelets. Computers \& structures, 84(10-11), 718-731.

Castro, L. M. S., \& de Freitas, J. A. T. (2001). Wavelets in hybrid-mixed stress elements. Computer Methods in Applied Mechanics and Engineering, 190(31), 3977-3998.

Cen, S., Fu, X. R., \& Zhou, M. J. (2011). 8-and 12-node plane hybrid stress-function elements immune to severely distorted mesh containing elements with concave shapes. Computer Methods in Applied Mechanics and Engineering, 200(29-32), 2321-2336. 
Cen, S., Zhou, M. J., \& Fu, X. R. (2011b). A 4-node hybrid stress-function (HS-F) plane element with drilling degrees of freedom less sensitive to severe mesh distortions. Computers \& Structures, 89(56), 517-528.

Clough, R. W., \& Wilson, E. L. (1999, August). Early finite element research at Berkeley. In Fifth US National Conference on Computational Mechanics (pp. 1-35).

Comi, C., \& Perego, U. (2001). Numerical aspects of nonlocal damage analyses. Revue européenne des éléments finis, 10(2-4), 227-242.

Comi, C., \& Perego, U. (2001). Symmetric and non-symmetric non-local damage formulations: an assessment of merits. In Proceedings of ECCM 2001 European conference on computational mechanics, (pp. 1-19). Cracow.

Cook, R. D. (2007). Concepts and applications of finite element analysis. John Wiley \& Sons.

Cottrell, J. A., Hughes, T. J., \& Bazilevs, Y. (2009). Isogeometric analysis: toward integration of CAD and FEA. John Wiley \& Sons.

De Almeida, J. M., \& De Freitas, J. T. (1991). Alternative approach to the formulation of hybrid equilibrium finite elements. Computers \& Structures, 40(4), 1043-1047.

De Almeida, J. M., \& Pereira, O. A. (1996). A set of hybrid equilibrium finite element models for the analysis of three-dimensional solids. International Journal for Numerical Methods in Engineering, 39(16), 2789-2802.

Teixeira de Freitas, J. A. (1990). Mixed and hybrid symmetric formulations for the boundary integral method. European journal of mechanics. A. Solids, 9(1), 1-20.

de Freitas, J. T. (1999). Hybrid finite element formulations for elastodynamic analysis in the frequency domain. International journal of solids and structures, 36(13), 1883-1923.

Freitas, J. A. T., Almeida, J. P. B. M., \& Pereira, E. M. B. R. (1993). Alternative hybrid formulations for the finite element method. In Proc. 7th World Congress Finite Element Method(pp. 264-271).

Freitas, J. A. T., Almeida, J. P. B. M., \& Pereira, E. M. B. R.(1996). Non-conventional formulations for the finite element method. Structural Engineering and Mechanics, 4, 655-678.

Freitas, J. A. T., Almeida, J. P. B. M., \& Pereira, E. M. B. R.(1999). Non-conventional formulations for the finite element method. Computational Mechanics, 23(1), 488-501.

Ghali, A., \& Nevillle, A. M. (1997). Structural Analysis: A unified Classic and Matrix Approach. London, E \& FN Spon.

Holzapfel, G. A.(2000). Nonlinear Solid Mechanics. Austria, Wiley and Sons LTD.

Hughes, T. J. R.(2003). The Finite Element Method, Linear Static and Dynamic Finite Element Analysis. Dover.

Jirousek, J., \& Leon, N. (1977). A powerful finite element for plate bending. Computer Methods in Applied Mechanics and Engineering, 12, 77-96.

Liu, G. R. (2003). Mesh Free Methods. USA, CRC Press.

Malkus, D. S., \& Hughes, T. J. R.(1978). Mixed finite element methods - Reduced and selective integration techniques: A unification of concepts. Computer Methods in Applied Mechanics and Engineering, 1(15), 63-81.

Mazars, J.(1984) J.. Application de la mécanique de l'endommagement au comportement non lineaire et à la rupture du béton de structure. Paris, Université Paris 6. Ph.D Thesis

Mendes, L. A. M., \& Castro, L. M. S. S.(2009) Hybrid-mixed stress finite element models in elastoplastic analysis. Finite Elements in Analysis and Design 45(12), 863-875.

Moes, N., Dolbow, J., \& Belytschko, T.(1999). A finite element method for crack growth without remeshing. International Journal for Numerical Methods in Engineering, 46, 131-150.

Pereira, E. M. B. R., \& Freitas, J. A. T. (1996a). A hybrid-mixed element model based on legendre polynomials for reissner-mindlin plates. Computer Methods in Applied Mechanics and Engineering 136(1-2), 111-126.

Pereira, E. M. B. R., \& Freitas, J. A. T.(1996b). A mixed-hybrid finite element model based on orthogonal functions. International Journal for Numerical Methods in Engineering, 39(8), 1295-1312.

Pereira, E. M. B. R., \& Freitas, J. A. T. (2000). Numerical Implementation of a Hybrid-Mixed Finite Element Model for Reissner-Mindlin Plates. Computer \& Structures 74(3), 323-334. 
Pereira, O. J. B. A.(1996). Utilização de Elementos Finitos de Equilíbrio em Refinamento Adaptativo. Lisbon, Instituto Superior Técnico. Ph.D Thesis

Pian, T. H. H., \& Tong, P. (1969). Basis of finite element methods for solid continua. International Journal for Numerical Methods in Engineering, 1, 3-28.

Ruoff, G.(1973). Finite Elemente in der Statik. ch. Die praktische Berechnung der Kombination der Trefftzschen Metode und bei flachen Schalen: 242-259.

Santos, H. A. F. A. (2009). Duality in the geometricall exact analysis of frame structures. Lisbon, Instituto Superior Técnico. Ph.D Thesis

Shephard, M. S., Niu, O., \& Baehmann, P. L.(1989). Some results using projectors for error indication and estimation. In Adaptive Methods for Partial Differential Equations, Philadelphia.

Silva, M. C., \& Castro, L. M. S. S. (2004). Hybrid-mixed stress formulation with continuum damage models continuum damage models. XXV CILAMCE. Recife, Gráfica Bagaço

Silva, M. C., \& Castro, L. M. S. S. (2006a). Hybrid-mixed stress formulation using continuum damage models. Communications in Numerical Methods in Engineering, 22, 605-617.

Silva, M. C., \& Castro, L. M. S. S.(2006b). Hybrid and mixed finite element formulations for softening materials. European Conference on Computational Mechanics - Solids, Structures and Coupled Problems in Engineering, Lisboa, ECCM.

Spiegel, R. M., \& Liu, J. (1999). Schaum's Mathematical Handbook of Formulas and Tables. New York, Schaum McGraw-Hill.

Stein, E. (1973). Finite Elemente in der Statik. ch. Die Kombination des modifizierten Treffzschen Verfahrens mit der Methode der Finiten Elemente: 172-185.

Veubeke, B. M. (1965). Displacement and Equilibrium models in the Finite Element Method. Stress Analysis: Wiley.

Zienkiewicz, O. C. (2000). Achievements and some unsolved problems of the finite element method. International Journal for Numerical Methods in Engineering, 47, 9-28.

Zienkiewicz, O. C., \& Taylor, R. L. (2000). The Finite Element Method: Basis. Londres, 1, B-H.

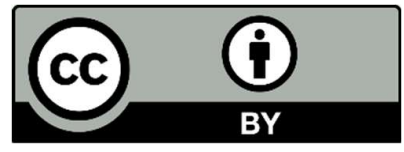

(C) 2019 by the authors; licensee Growing Science, Canada. This is an open access article distributed under the terms and conditions of the Creative Commons Attribution (CC-BY) license (http://creativecommons.org/licenses/by/4.0/). 